\title{
Was Laxanzienabusus anrichten kann
}

\begin{abstract}
Bei einem 67-jährigen Mann bestanden seit zwei Monaten Bauchschmerzen, Übelkeit und eine Obstipation. In den letzten zwei Wochen war eine Spannung der Bauchdecken dazugekommen. Der Patient benutzte seit vielen Jahren verschiedene Laxanzien.
\end{abstract}

- Die Vitalparameter bei der Erstuntersuchung waren unauffällig, der Patient machte aber einen ängstlich-agitierten Eindruck. Die Darmgeräusche waren sehr träge, bei der Palpation bemerkte man eine derbe, nicht bewegliche, schmerzhafte Raumforderung im linken unteren Quadranten. Das CT des Abdomens ergab ein riesiges Fäkalom im kleinen Becken und im Bereich des linken unteren Abdomens.

Der Versuch, den impaktierten Stuhl mittels Einlauf oder Sigmoidoskopie zu verflüssigen, gelang nicht. Man führte schließlich eine mediane Laparotomie
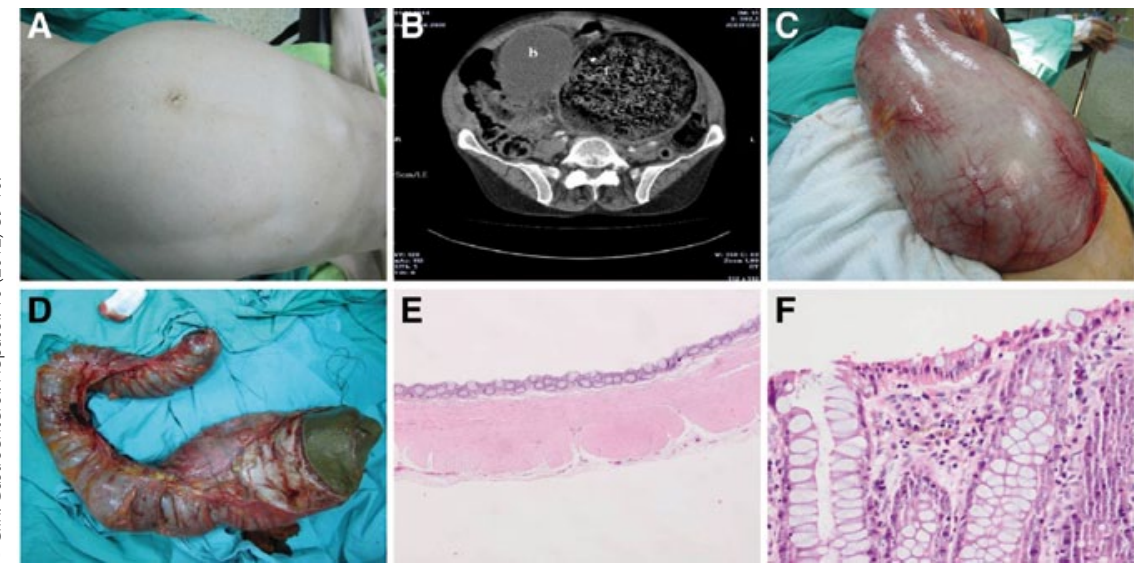

Massives Fäkalom präoperativ, im CT und Operations- sowie histologischem Präparat.

durch. Aufgrund der Größe des Fäkaloms musste der Schnitt vom Xiphoid bis zur Symphyse geführt werden. Der Durchmesser des Sigmas betrug etwa $20 \mathrm{~cm}$.

\section{Kommentar}

Fäkalome treten am häufigsten im distalen Kolon und Rektum auf. In sehr fortgeschrittenen Fällen und bei besonders hart eingedick- tem Stuhl ist eine chirurgische Entfernung erforderlich. Bei dem Patienten wurde im Resektat eine Melanosis coli festgestellt, ein Befund, der für den langjährigen Gebrauch von Anthrachinon-haltigen Laxanzien sprach.

H. S. FÜESSL .

- A.F. Yucel et al.

A giant abdominal mass: fecaloma. Clin. Gastroenterol. Hepatol. 10 (2012) e9-10.

\section{Chronische Kreuzschmerzen: Kortison hilft nicht}

Seit mehr als 30 Jahren werden epidurale Steroidinjektionen zur Behandlung von radikulären Schmerzen eingesetzt, obwohl der wissenschaftliche Beweis der Wirksamkeit aussteht.

- In einer prospektiven, multizentrischen, doppelblinden, randomisierten, kontrollierten Studie aus Norwegen wurden zwischen Oktober 2005 und Februar 2009461 Patienten gescreent, die seit mehr als zwölf Wochen unter chronischen lumboradikulären Schmerzen litten. Davon wurden 133 Patienten randomisiert und in drei Gruppen eingeteilt: Die erste Gruppe erhielt eine subkutane Scheininjektion von $2 \mathrm{ml}$ Kochsalzlösung, die zweite Gruppe eine kaudale epidurale Injektion von $30 \mathrm{ml}$ Kochsalzlösung und die dritte Gruppe eine kaudale epidurale Injektion von 40 mg Triamcinolon.
Die Injektionen wurden zweimal im Abstand von zwei Wochen durchgeführt. 40 Patienten fanden sich in der Scheininjektionsgruppe, 39 in der Kochsalzlösungsgruppe und 37 in der Steroidgruppe. Die mittlere Dauer der Krankschreibung aufgrund der Beschwerden betrug zwischen 14 und 21 Wochen. Die MRT zeigte bei zwei Drittel der Patienten eine Bandscheibenprotrusion.

\section{Kommentar}

Es ist ein großer Verdienst der Arbeitsgruppe aus Norwegen, zu dieser schmerztherapeutisch sehr umstrittenen Fragestellung jetzt eine vernünftige randomisierte und placebokontrollierte Studie durchgeführt zu haben. In früheren Studien war immer wieder kritisiert worden, dass nicht völlig ausgeschlossen werden kann, dass die Injektionen von $20 \mathrm{ml}$ physiologischer Kochsalzlösung eine biologische Wirkung haben könnten. Daher haben die Autoren in der
Weder für den primären Endpunkt noch für die sekundären Endpunkte ergab sich nach sechs, zwölf und 52 Wochen ein Unterschied zwischen den drei Behandlungsgruppen. Epidurale Kortisoninjektionen sind also bei chronisch lumboradikulären Schmerzen nicht wirksam. 